\title{
Comparative study of AIN dielectric films' electrical properties for MEMS capacitive switches
}

\author{
M. Koutsoureli ${ }^{1 *}$, A. Adikimenakis ${ }^{2}$, L. Michalas ${ }^{1}$, E. Papandreou ${ }^{1}$, G. Stavrinidis ${ }^{2}$, G. \\ Konstantinidis $^{2}$, A. Georgakilas ${ }^{2}$ and G. Papaioannou ${ }^{1}$ \\ ${ }^{1}$ Solid State Physics Section, University of Athens, GR-15784, Athens, Greece \\ ${ }^{2}$ IESL -FORTH, GR-71110, Heraklion, Greece \\ * Corresponding author: mkoutsoureli@phys.uoa.gr
}

Aluminum nitride (AlN) piezoelectric thin films are very popular in RF micro-machined resonators and filters MEMS devices. The advantages of these films arise from their high resistivity and piezoelectric coefficient, which is the largest among nitrides, as well as the possibility of being deposited at temperatures lower than $500^{\circ} \mathrm{C}$ and patterned using conventional photolithographic techniques. AlN generally exhibits smaller piezoelectric and dielectric constants and differs from PZT materials in that it is polar rather than ferroelectric. Different techniques such as sputtering, metal-organic chemical vapor deposition (MOCVD), pulsed laser deposition (PLD), plasma enhanced chemical vapor deposition (PECVD) and molecular beam epitaxy (MBE) have been used so far in order to investigate and improve the properties of AIN films.

The present paper investigates and compares the electrical properties of aluminum nitride films deposited by different techniques. Metal-Insulator-Metal (MIM) capacitors with AlN films have been grown by magnetron sputtering and plasma - assisted molecular beam epitaxy (PAMBE) methods. The sputtered AlN films were $\mathrm{N}$ face with a thickness of $200 \mathrm{~nm}$ while PA-MBE grown films had a thickness of $100 \mathrm{~nm}$. The assessment is based on electrical characterization measurements obtained under vacuum and in a temperature range from 300 to $400 \mathrm{~K}$.

The experimental data revealed the dominant conduction mechanism of the AlN films and it has been found that charge collection takes place through variable range hopping in the magnetron sputtered film while Poole-Frenkel effects dominate the conduction process in PAMBE grown films. Moreover, charging and discharging processes have been found to obey stretched exponential law, in agreement to Kohlrausch-Williams-Watts polarization's relaxation, found in many disordered materials.

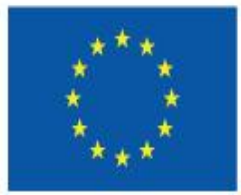

European Union European Social Fund

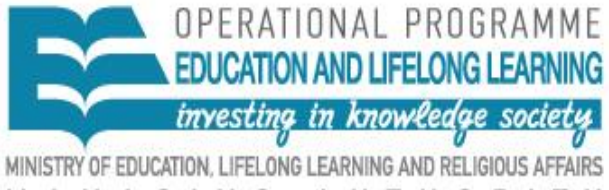

MAN A G I N G A U THORITY

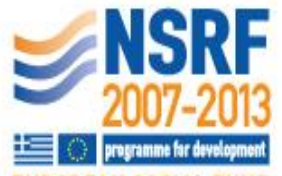

EUROPEAN SOCIAL FUND Co-financed by Greece and the European Union 